\title{
Como os equinos percebem seus predadores? Uma visão evolucionista e prática
}

\section{How do equines perceive their predators? An evolutionary and practical view}

\author{
Pedro Henrique Esteves Trindade ${ }^{1,2}$, Franciely de Oliveira Costa ${ }^{2,3^{*}}$ \\ ${ }^{1}$ Faculdade de Medicina Veterinária e Zootecnia (FMVZ), Universidade Estadual Paulista (UNESP), Botucatu, SP, Brasil \\ ${ }^{2}$ Grupo de Estudos e Pesquisas em Etologia e Ecologia Animal (ETCO), Universidade Estadual Paulista (UNESP), Jaboticabal, SP, Brasil \\ ${ }^{3}$ Faculdade de Ciências Agrárias e Veterinárias (FCAV), Universidade Estadual Paulista (UNESP), Jaboticabal, SP, Brasil
}

\section{Resumo}

São diversas as circunstâncias em que os cavalos são acometidos por emoções negativas como medo ou ansiedade, podendo resultar em reações defensivas explosivas, que possivelmente podem derivar em acidentes. Estas respostas de defesa fazem parte de um conjunto de comportamentos classificados como inatos, os quais foram desenvolvidos e aprimorados ao longo do processo evolutivo do Equus caballus na tentativa de sobreviver às investidas de caça dos predadores, contribuindo para a preservação da espécie. Apesar de o cavalo doméstico, que convive habitualmente com o ser humano, já não ter contato regular com predadores naturais, os mecanismos defensivos e as emoções negativas que envolvem tal situação ainda permanecem ativos, produzindo respostas comportamentais específicas. Torna-se relevante, portanto, o estudo aprofundado sobre quais elementos desencadeiam tais reações defensivas nos cavalos a fim de que as causas desses comportamentos sejam evitadas, reduzindo, assim, o número de acidentes. Por isso, o objetivo dessa revisão foi investigar como os equinos percebem seus predadores, as possíveis hipóteses evolutivas, bem como suas implicações práticas. Este tema, quando melhor esclarecido, poderá contribuir para o aperfeiçoamento de instalações e manejos relacionados aos equinos, com a intenção de reduzir ou ainda evitar tais reações de medo, que implicam no empobrecimento do bem-estar animal.

Palavras-chave: Bem-estar animal. Comportamento inato. Emoções negativas. Medo. Teste de novo objeto.

\begin{abstract}
There are several circumstances in which horses are affected by negative emotions such as fear or anxiety, which can result in explosive defensive reactions that may possibly lead to accidents. These defense responses are part of a set of behaviors classified as innate, which were developed and improved throughout the evolutionary process of Equus caballus in an attempt to survive predation hunts, contributing to the preservation of the species. Although
\end{abstract}


the domestic horse, which habitually coexists with humans, no longer have regular contact with natural predators, the defensive mechanisms and negative emotions that involve such a situation still remain active, producing specific behavioral responses. Therefore, it is relevant to study in depth what elements trigger such defensive reactions in horses, so that the causes of these behaviors are avoided, thus reducing the number of accidents. Therefore, the objective of this review was to investigate how equines perceive their predators, possible evolutionary hypotheses, as well as their practical implications. This subject, when clarified, may contribute to the improvement of facilities and management related to equines, with the intention of reducing or even avoiding such reactions of fear, which imply the impoverishment of animal welfare.

Keywords: Animal welfare. Innate behavior. Negative emotions. Fear. Novel object test.

\section{Introdução}

Mundo afora, os cavalos vêm sendo envolvidos em várias atividades equestres como competições esportivas, reprodução, lazer, companhia, uso militar, terapêutico e agropecuário. O Brasil, particularmente, tem o quarto maior rebanho mundial de equinos, estimado em 5 milhões de cabeças (Lima e Cintra, 2016). Tal interação humano-animal pode derivar em situações que os cavalos são acometidos pelas sensações de medo ou ansiedade, podendo desencadear reações defensivas como uma fuga abrupta, possivelmente resultando em lesões tanto para os animais como para os manejadores. 0 medo é geralmente definido como uma reação à percepção de um perigo real, enquanto a ansiedade é definida como a reação a um potencial perigo que ameace a integridade do indivíduo (Forkman et al., 2007; Beauchamp, 2017).

Para os animais em vida livre, essas reações defensivas são muito importantes para a preservação da vida e da própria espécie, como forma de evitar ao máximo fontes de perigo (predadores, por exemplo) (Forkman et al., 2007; Beauchamp, 2017). Estas reações defensivas fazem parte de um conjunto de comportamentos classificados como inatos, os quais foram aprimorados de acordo com as pressões ambientais ao longo do processo evolutivo do Equus caballus, na tentativa de sobreviver às investidas de caça dos predadores (Hanggi e Ingersoll, 2012). Os comportamentos inatos são caracterizados como comportamentos motivados, pois sua exibição depende da combinação de estímulos internos e externos ao animal (Paranhos da Costa, 2002). Todavia, previamente à ação de fuga, a presa encontra pistas dos seus predadores, pois o sucesso da presa depende da capacidade de conhecer a localização precisa do seu predador para poder escapar antes mesmo que o ataque ocorra (Heffner e Heffner, 1984). As pistas representam potencial fonte de informação que, segundo Hauser (1996), diferem dos sinais por dois aspectos: (i) as pistas tendem a permanecer ligadas, enquanto os sinais são mais plasmáticos e podem estar ligados ou desligados; (ii) os sinais, diferente das pistas, são produzidos como resposta a alterações socioecológicas (socioecologically) relevantes, que são temporárias no ambiente. Por isso, as presas evoluíram para conhecer as pistas de seus predadores, na tentativa de evitá-los; como um valor adaptativo da espécie.

Apesar de os animais em cativeiro não terem contato regular com predadores naturais, os mecanismos defensivos e as emoções negativas que envolvem tal situação ainda persistem, aptos a produzir respostas comportamentais específicas quando estimulados (Forkman et al., 2007). Tal fato se materializa pelos achados de Keeling et al. (1999), nos quais foram apontadas as reações de medo dos cavalos como representativas de $27 \%$ das causas de acidentes envolvendo equinos e seres humanos. Portanto, em face dessas informações já documentadas, torna-se relevante o estudo aprofundado sobre quais elementos estimulam reações defensivas nos cavalos, a fim de que suas causas sejam evitadas, ou ao menos reduzidas, e que o número de acidentes e seus efeitos deletérios diminuam (von Borstel et al., 2010).

Para tanto, o entendimento de como os cavalos percebem as pistas de seus predadores se revela fundamental. Entretanto, o conhecimento científico contemporâneo ainda é restrito no que tange o modo como este processo ocorre integralmente. Este tema, quando melhor esclarecido, poderá ter 
implicações práticas para aprimorar as instalações e os manejos que envolvem os equinos, com a intenção de diminuir ou ainda evitar tais reações de medo, que reduzem o bem-estar animal. Por isso, o objetivo dessa revisão foi investigar como os equinos percebem seus predadores, as possíveis hipóteses evolutivas, bem como suas implicações práticas.

\section{Sentido olfativo}

O sistema olfativo dos equinos pode ser considerado como bastante eficiente. Alguns autores (Waring, 2003; Mills e Nankervis, 2005) relataram que os equinos utilizam a comunicação química e, por consequência, o sentido da olfação para diversos processos de comunicação como, por exemplo, a identificação individual, coordenação, espaço individual e de grupos, comunicação entre égua e potro, orientação, interesse sexual e sinais de alarme.

Christensen e Rundgren (2008) conduziram três experimentos sobre percepção de odores de predadores por cavalos. Estes experimentos foram feitos em arenas com paredes de 2,4 m de altura e cocho de concentrado localizado no lado oposto da porta de entrada. A priori, 45 cavalos foram habituados ao recinto e alimentados no cocho, com milho e melaço. Os cientistas investigaram como os cavalos respondiam a diferentes estímulos, como: (i) urina de lobos, leões e cavalos; (ii) sangue de cavalos no pré-abate e derivado de pele de lobo; e, por fim, (iii) estímulo auditivo repentino (barulho produzido por sacola plástica) na presença ou ausência de derivado de pele de lobo. Todos os estímulos odoríferos, em seus respectivos ensaios, foram esfregados no cocho (sem contaminação por alimento) e no piso do recinto (areia ou serragem) ao redor do cocho. Já o estímulo auditivo repentino foi executado do lado de fora da arena na direção de trás do cocho. A posteriori da introdução dos estímulos, os equinos foram equipados com um marcador de frequência cardíaca digital (Polar s810i) e conduzidos para dentro da arena, tendo sido filmados por $2 \mathrm{~min}$.

Os resultados do primeiro experimento de Christensen e Rundgren (2008) mostraram que não houve diferença significativa no tempo gasto pelos cavalos em cheirar a urina de predadores e a de outros cavalos. Quando expostos ao sangue de cavalos no pré-abate, no segundo experimento, os equinos apresentaram mudança significativa de comportamento, exibindo estado de vigilância e maior tempo cheirando, apesar de a frequência cardíaca não ter aumentado. No terceiro experimento, o estímulo odorífero aliado com o estímulo auditivo repentino promoveu aumento da frequência cardíaca dos cavalos em comparação com apenas o estímulo odorífero. Diante desses resultados, os autores concluíram que o odor de predadores não amedrontou os cavalos, exceto quando acrescidos de um estímulo auditivo repentino.

A partir dos resultados obtidos por Christensen e Rundgren (2008), não é possível determinar se os equinos são capazes de identificar o odor de predadores. Seria necessário conhecer as experiências prévias desses equinos, a fim de identificar se algum deles já teria sido exposto ao odor de um predador, uma vez que não é conhecido se esse tipo de reconhecimento seria inato ou aprendido. Nesse contexto, os cavalos do experimento poderiam não ter tido nenhum tipo de associação prévia entre o odor de urina de lobos ou leões e uma experiência negativa, não apresentando, portanto, qualquer reação comportamental que diferenciasse a resposta perante a urina de cavalos. Independentemente disso, apesar de não se notar diferença comportamental dos equinos frente à urina de predadores e de outros cavalos, podese dizer que os cavalos perceberam os estímulos odoríferos testados, visto que demonstraram curiosidade diante das urinas, supondo que o sistema olfativo é suficientemente sensível para detectar tais odores. Além disso, o estímulo odorífero associado a um estímulo auditivo talvez se apresente como uma pista mais amedrontadora, sendo um indício de que o equino apresentaria um sistema auditivo mais sensível que o olfativo para percepção da pista de predadores.

Para Christensen e Rundgren (2008), os cavalos foram selecionados para não se amedrontarem com odores dos predadores pelo fato de conviverem em um mesmo ambiente; desta forma, economizam energia, evitando o estado constante de alerta 
ou vigília (resposta de "luta ou fuga"). Apesar dessas pressuposições em relação aos equinos, comportamentos indicativos de medo foram observados em ratos, ratazanas, cervos, coelhos, golfinhos, ouriços, ovinos e bovinos quando expostos à urina e fezes de seus predadores naturais (Pfister et al., 1990; Arnould e Signoret, 1993; Arnould et al., 1993; Terlouw et al., 1998; Apfelbach et al., 2005).

Avigilância se refere ao estado ouà ação de manter uma atenção cuidadosa ("concentração"), mediante a utilização dos cinco sentidos combinados para identificar um perigo em potencial (Beauchamp, 2017). Estudos recentes indicaram que gaivotas, antrazes (Beauchamp, 2009), garças (Ge et al., 2011), veados (Pays et al., 2007) e javalis (Podgórsk et al., 2016) coordenam e sincronizam o estado de vigilância dentro do grupo. Assim, os animais localizadosnas bordas do grupo realizam avigilância, enquanto os animais dentro do grupo permanecem relaxados. Neste sentido, os cientistas afirmam haver um revezamento entre os animais das bordas (em vigilância) com aqueles que se encontram no centro do grupo (relaxados). Michelena et al. (2012) observaram que grupos menores de ovelhas apresentaram maiores taxas de cortisol salivar em comparação a grupos mais numerosos. Desta forma, para os animais considerados na classe de presas, a quantidade de indivíduos no grupo pode influenciar na taxa de vigilância e, por consequência, nos hormônios relacionados à resposta de "luta ou fuga" (Beauchamp, 2017). Assim, é possível supor que não seria possível manter a vigilância sem a cooperação de um grupo.

Se adotarmos a hipótese de que os equinos não se amedrontam com odores de predadores, dois questionamentos nos saltam aos olhos: (1) quais foram as pressões ambientais que selecionaram - Equus caballus quanto a sua percepção de predadores? (2) Quais foram as pressões ambientais enfrentadas pelos outros mamíferos que são mais sensíveis a odores de seus predadores? É possível que a explicação para estas indagações esteja, subliminarmente, nas características dos predadores, no ambiente e nas características específicas de cada presa. Para responder a tais questões, faz-se necessário um detalhado e aprofundado estudo sobre o tema em discussão, em especial estudos por métodos comparativos
(Hauser, 1996), analisando a homoplasia entre espécies homólogas e levando em conta as pressões ambientais de cada espécie.

Retomando a discussão mais ampla sobre como os equinos percebem seus predadores, Christensen et al. (2005) demonstraram que cavalos domésticos reagem mais a estímulos auditivos e visuais em comparação a estímulos olfatórios (óleo de eucalipto). No entanto, o estímulo olfatório utilizado nesse estudo não foi um odor do meio natural ao qual os equinos deveriam apresentar uma resposta aversiva. Por isso, é muito provável que estudos futuros focados na percepção de odores de predadores por cavalos possam responder com maior profundidade esses questionamentos.

Aparentemente, em uma escala de relevância para os equinos, os estímulos olfatórios poderiam ser classificados como secundários para a percepção das pistas dos predadores. É óbvio que os odores não podem e nem devem passar despercebidos pelos cavalos, uma vez que eles têm um sistema olfativo eficiente. No entanto, os estímulos odoríferos representam uma percepção precoce do predador, não necessariamente correspondendo à presença do predador no imediato reconhecimento do odor, pois ele pode apenas ter passado por aquela região. De modo oposto, os estímulos sonoros de um predador obrigatoriamente representam perigo eminente pela presença in loco ou nas proximidades, podendo, desta forma, representar maior prioridade de preocupação. Assim sendo, a interrogativa recorrente remete à seguinte reflexão: a audição seria o principal sentido de percepção das pistas dos predadores por parte dos equinos?

\section{Sentido auditivo}

A capacidade auditiva de um animal depende de quatro fatores: ser presa ou predador, o tipo de habitat que ocupa, se o animal é preferencialmente mais ativo durante o dia ou à noite e, por fim, as capacidades visuais do animal (Heffner e Heffner, 1984). No ambiente natural, o êxito do predador está em saber com exatidão onde está sua presa para caçá-la, enquanto o sucesso da presa está em conhecer a localização precisa do seu predador para poder escapar. Esta hipótese é sustentada pela 
alta acuidade auditiva de gatos e cães (predadores) e baixa acuidade auditiva de cavalos e roedores (presas). Em contrapartida, Boyd e Keiper (2005) relataram que cavalos adultos dificilmente são predados e que os ataques ocorrem com maior frequência a animais jovens ou doentes. É possível que esta relação não seja assim tão simplista a ponto de ser respondida apenas com o fator da capacidade auditiva do animal, como sugerida por Heffner e Heffner (1983).

0 ambiente também exerce grande influência na seleção das espécies, sendo determinante na prevalência do sentido mais adequado à manutenção da espécie. Dessa maneira, em espécies com habitat em florestas densas e com baixa visibilidade, a audição é de extrema valia para a sobrevivência. Por outro lado, para animais que vivem em planícies, a visão deve ser mais importante (Heffner e Heffner, 1984). Talvez seja por esta pressão ambiental que o Equus caballus não tenha uma boa acuidade auditiva.

Em relação ao ciclo circadiano dos animais (noturnos ou diurnos), sabe-se que em animais noturnos a visão é menos útil, por isso, estes podem obter vantagens se tiverem uma boa capacidade auditiva. Essa hipótese, porém, pode ser contestada, uma vez que elefantes são adaptados tanto para ambientes claros como para escuros e não têm baixa capacidade auditiva, bem como existem animais noturnos com baixa capacidade auditiva, como o ouriço e algumas espécies de ratos (Heffner e Heffner, 1984).

$\mathrm{Na}$ busca pelo entendimento das capacidades auditivas dos equinos, Heffner e Heffner (1984) conduziram um experimento para avaliar a capacidade dos equinos em localizar um som (pista) e em diferenciar a fonte do som pelo próprio som (o que é), e concluíram que os cavalos não têm boa capacidade para isso. Os autores afirmaram que os equinos têm maior facilidade de localização do som e diferenciação da fonte sonora pelo próprio som quando conseguem visualizar o objeto com a visão binocular, ou seja, olhando o objeto de frente. Esta pesquisa nos conduz à reflexão de que a audição talvez não seja tão relevante para os equinos perceberem as pistas de seus predadores, como sugerido por Christensen et al. (2005) e Christensen e Rundgren (2008). Porém, admite-se que a audição tenha sua parcela de contribuição na percepção de pistas de predadores ou outras funções dentro do grupo e entre grupos próximos.

Para evitar a atração de predadores, os equinos frequentemente se comunicam sem utilizar a vocalização (McGreevy, 2004). Ademais, é de se ponderar que a comunicação acústica (estabelecida pela emissão de sons) é energeticamente mais custosa quando comparada à linguagem corporal (Prestwich et al., 1989; Deecke et al., 2005). Todavia, os equinos emitem sons sutis de forma incidental durante a inalação, que ocorrem em duas circunstâncias: previamente à emissão de um sinal de alarme ou blow, sendo este mais rápido (0,3 - 0,5 s) (Waring, 1971, 2003), e também quando um cavalo em decúbito faz uma respiração forçada, desta vez sendo mais demorada $(1,0$ 1,8 segundos). Além do mais, os equinos emitem vários tipos de vocalizações (relincho, guinchos, gemidos, roncos, lábios batendo, entre outros) que variam de acordo com a motivação do animal, o sexo, o tamanho do corpo, a identidade individual e o contexto do emissor (Yeon, 2012), fatos estes que explicitam a finalidade da capacidade auditiva dos equinos que, pelo que se apresenta, não foi em essência selecionada para ser um "scanner de ondas sonoras" (Heffner e Heffner, 1984).

Com as evidências apresentadas até esta etapa da revisão, é possível considerar que a olfação e a audição não representam um dos principais sentidos para identificação dos predadores. Desta forma, é natural pensar que os estímulos visuais sejam os de maior relevância para os cavalos no processo de reconhecimento de seus predadores. Mesmo assim, é de se indagar: a visão seria realmente o sentido de maior capacidade dos cavalos para percepção de seus predadores? Quais as perguntas científicas que os experimentos dispostos na literatura poderiam responder a propósito deste tema?

\section{Sentido visual}

Aplicando a teoria da evolução, para sustentar a hipótese de que os cavalos foram selecionados para perceber seus predadores pela visão, é preciso desenvolver ensaios experimentais baseados nas quatro questões de Timbergen (Hauser, 1996). No entanto, experimentos deste gênero podem 
demandar muitos anos. Assim, de posse das conclusões de Charlies Robert Darwin e Alfred Russel Wallace de que os comportamentos são modulados pela variabilidade, hereditariedade, competição, seleção natural e adaptação (Mills e Nankervis, 2005), já podemos empiricamente adotar uma possível hipótese para a evolução de um dado comportamento.

Retomando a discussão em sua maior amplitude, como pressuposto por Heffner e Heffner (1984), os equinos podem efetivamente ter sido selecionados para perceber seus predadores pela visão, visto que tinham como seu habitat natural a vida em grandes planícies. Em consonância a esta hipótese, os resultados científicos de Hanggi e Ingersoll (2012) demonstraram que os equinos conseguem identificar diversos objetos a partir de vários ângulos da sua visão monocular (imagem formada a partir da luminosidade captada por apenas um olho), contrapondo o que era convencionado por outros autores (Heffner e Heffner, 1984; Hanggi, 1999), que afirmavam que os equinos apenas distinguiam objetos com a visão binocular. Ademais, Hanggi e Ingersoll (2012) consideram que os equinos se amedrontam com maior frequência quando submetidos a duas situações: em ambientes desconhecidos e quando neste meio apresentamse barulhos ou objetos surgindo repentinamente. Situações como estas podem ocasionar grande pânico e instigar o cavalo a um comportamento inato de fuga a qualquer custo.

Ensaios básicos de discriminação têm demonstrado que o sucesso dos cavalos em reconhecer e diferenciar objetos pode estar relacionado com a altura em que estejam posicionados. As habilidades perceptivas apresentam taxas de sucesso mais elevadas quando os estímulos são posicionados ao nível dos olhos ou abaixo deles (Gardner, 1937; Hanggi e Ingersoll, 2012), e quando são colocados no chão, os equinos são mais bem-sucedidos (Hall, 2007). Esses resultados corroboram com a hipótese de que os cavalos estão adaptados para permanecer pastando por grande parte do tempo e vigilantes aos estímulos visuais representando potenciais perigos ao seu entorno (Mills e Nankervis, 2005).

Os equinos possuem os olhos posicionados lateralmente no crânio, de modo a lhes proporcionar uma visão panorâmica ao redor de seu corpo. É possível que o campo de visão monocular dos equinos seja de 190 a 195 graus no eixo horizontal e 178 graus no eixo vertical quando o cavalo estiver olhando para um ponto fixo. Quando as imagens monoculares produzidas pelos dois olhos são combinadas, o campo visual horizontal tem uma amplitude de até 350 graus, restando apenas alguns pontos cegos (Harman et al., 1999; Mills e Nankervis, 2005; Miller e Murphy, 2010).

Sob a ótica evolucionista, é possível crer que a visão dos equinos tinha grande valia durante a vida em liberdade pelas planícies que o Equus caballus habitava, especialmente para a identificação de predadores que se aproximavam do grupo. Ademais, fisiologicamente, o sistema visual dos cavalos é bastante desenvolvido e adaptado para uma visão panorâmica do que está acontecendo ao entorno do seu corpo. Tais evidências se mostram como fortes indícios para supor que a visão seja o sentido mais desenvolvido pelos equinos para a percepção dos seus predadores. Apesar disso, não se pode desconsiderar a contribuição do olfato e da audição na percepção dos predadores e suas pistas. Estes vestígios, como até então evidenciados nesta revisão, suscitam novo questionamento: como os conhecimentos apresentados podem interferir na rotina de um cavalo e na prática de manejo por seu tutor nos dias atuais?

\section{Implicações práticas}

Não são raros os casos em que cavalos submetidos a situações estressantes despertam uma motivação interna resultante em reações defensivas explosivas como, por exemplo, uma fuga abrupta, esquivar-se de um objeto ou animal em particular, debater-se durante tentativa de contenção física, dentre outras. Também não é incomum que eventos dessa magnitude acabem por ocasionar acidentes com danos e até lesões tanto no animal quanto no tutor, cavaleiro ou cuidador do cavalo. Keeling et al. (1999) mostraram que $27 \%$ das causas de acidentes envolvendo equinos e humanos ocorrem por reações de medo dos cavalos. Como já esclarecido anteriormente, essas reações defensivas acontecem como produto de um 
comportamento inato, sempre na tentativa de evitar o desconhecido que supostamente representa perigo ao equino (Paranhos da Costa, 2002; Hanggi e Ingersoll, 2012).

Levando em conta que os cavalos têm grandes chances de apresentar reações de medo frente a um objeto, humano ou manejo desconhecido, determinadas condutas das pessoas envolvidas no seu manejo poderiam evitar acidentes. Por exemplo, a repetida exposição de um cavalo a um novo estímulo pode ocasionar habituação, diminuindo as reações de medo a este. A habituação é definida como o desaparecimento relativamente permanente de uma resposta resultante de uma estimulação repetida, que não é seguida por qualquer tipo de reforço (Sato, 1995). Isso foi caracterizado pelos resultados de Leiner e Fendt (2011), que avaliaram o processo de habituação de garanhões a dois tipos de novos objetos (atravessar uma lona e deparar-se com um guarda-chuva que abre próximo ao animal) representando estímulos visuais e auditivos. Os autores relataram que após o treinamento de habituação para um determinado objeto, a resposta de medo foi especificamente atenuada, enquanto a resposta de medo para o outro objeto (excluído do treinamento) permaneceu inalterada. Apesar disso, em estudo conduzido por Christensen et al. (2008b), onde garanhões foram habituados a seis diferentes objetos, os autores observaram que um alto grau de similaridade entre os objetos (por exemplo, coloração idêntica) parece ser crucial para os cavalos generalizarem a resposta frente a eles.

A habituação pode ser realizada para vários manejos rotineiros que particularmente representem algum tipo de barreira para o cavalo, como, por exemplo, escovar o topete, embarcar em um trailer ou caminhão, passar por bandeiras durante uma competição equestre, dentre outros. Para equinos demasiadamente amedrontados, é recomendável que o processo de habituação seja realizado com a inclusão de outro indivíduo, como foi caracterizado pelo estudo de Christensen et al. (2008a). Os resultados deste estudo mostraram que na presença de um cavalo habituado à exposição a um novo objeto específico, houve redução das reações de medo de um outro cavalo não habituado, confirmando o efeito social positivo na reação à exposição a novos objetos. Além disso, outro estudo relatou que quatro sessões de 50 min de manejo tradicional (escovação, levantar membros e arreamento do animal) melhoraram a relação humano-animal em animais ainda não domados (Fureix et al., 2009).

Outro comportamento aprendido que pode ser usado na prática é a estampagem (imprinting), realizado especificamente logo após o nascimento. o imprinting é um processo pelo qual os animais neonatos estabelecem uma preferência social por sua mãe (ou outro animal) durante um período crítico de desenvolvimento após o nascimento (Lorenz, 1965; Hess, 1972). Potros manejados imediatamente após o nascimento, por exemplo, podem apresentar menor irritação e maior facilidade de manejo em comparação com potros que não tiveram contato pós-natal imediato com humanos (Miller, 1991; Spier et al., 2004). Em estudo recente, esse processo de imprinting foi chamado de "sessão de pré-doma" e consistiu na estimulação tátil (massagem) de potros recémnascidos por $10 \mathrm{~min}$, diariamente, durante 15 dias, iniciando após o nascimento. É válido ressaltar que a sessão de pré-doma só era iniciada depois de o potro ter mamado o colostro e ocorria logo após o manejo regular de cura de umbigo. Após quatro meses, quatro avaliadores aplicaram o teste de aproximação forçada nos potros, sendo que os animais apresentaram generalização aos estímulos recebidos pelos humanos, exibindo respostas positivas inclusive com seres humanos não familiares. Tais resultados evidenciam que a adoção das boas práticas de manejo na fase inicial da vida dos potros favoreceu o estabelecimento de vínculos positivos com o ser humano (Schmidek et al., 2018).

Além do processo de habituação, também é possível utilizar o condicionamento operante, o qual consiste "no fornecimento de uma resposta ativa (operante) pela apresentação de um reforço (recompensa) se, e somente se, a resposta acontecer" (Paranhos da Costa, 2002). Trindade et al. (2016b), por exemplo, desenvolveram protocolo para condicionamento operante com reforço positivo alimentar para coletar amostras de sangue em cavalos. Esse protocolo foi aplicado por seis dias, com sessões de $20 \mathrm{~min}$, e os cavalos foram considerados adaptados pelo nível plasmático de cortisol, que estava dentro dos limites de referência. 
Já Warren-Smith e McGreevy (2007) aplicaram reforço positivo em cavalos para treiná-los a parar durante equitação com rédeas longas durante $4 \mathrm{a}$ 6 dias, em sessões de 30 min, e também obtiveram sucesso no treinamento. Nestes casos, tanto o processo de habituação como o de condicionamento operante visam treinar os animais, evitando que expressem as reações defensivas.

No estudo conduzido por von Borstel et al. (2010), cavalos de duas raças (Swedish Warmblood e Hanoverian) foram submetidos a um teste de reatividade, mediante a utilização de exposição a estímulos visuais e auditivos. Os autores concluíram que possivelmente as reações de medo podem ter bases genéticas, uma vez que houve diferença entre as raças e, por isso, sugeriram iniciar uma seleção genética com base na reatividade dos cavalos.

Neste contexto, para avaliar a reatividade de cavalos diante de estímulos desconhecidos, Trindade et al. (2016a) realizaram estudo preliminar aplicando um teste de novo objeto, que consistiu na apresentação de cinco novos objetos. Os cavalos foram conduzidos, montados por um cavaleiro não familiar, nos seguintes novos objetos: (1) passar entre duas bolas de $60 \mathrm{~cm}$ de diâmetro (uma de cor cinza e outra verde-clara); (2) passar por cima de uma lona azul, em forma de tapete; (3) passar em um corredor feito de cones verdefluorescente e fechamentos laterais vermelhos; (4) passar ao lado da metade de um barril branco de $200 \mathrm{~L}$; (5) e por fim, uma bola (verde-clara) de $60 \mathrm{~cm}$ de diâmetro foi rolada na direção do cavalo parado. Os animais foram avaliados antes e após uma cavalgada de $1000 \mathrm{~km}$, porém a cavalgada não teve efeitos expressivos na reatividade dos cavalos. Tal resultado sugere que as respostas comportamentais de medo não podem ser facilmente moduladas. Isso corrobora os achados de Leiner e Fendt (2011) de que o treinamento para um determinado estímulo não pode ser generalizado a outros estímulos. Possivelmente existe um componente genético influenciando as respostas de medo dos cavalos, como sugerido por von Borstel et al. (2010).

Os estudos supracitados evidenciam a relevância prática das reações comportamentais de medo. Ademais, como visto nas sessões prévias desta revisão, em uma escala de importância, os estímulos visuais parecem ter maior relevância para os cavalos, seguido dos estímulos auditivos e olfatórios. Desta forma, é preciso considerar o ambiente que os equinos estão alojados, assim como os manejos a que são submetidos para que evitem despertar reações defensivas explosivas. Quando não for possível prevenir a exposição dos animais a esses estímulos, é aconselhável aplicar práticas de manejo com o objetivo de reduzir essas respostas defensivas, como apresentado nesta última sessão.

\section{Conclusão}

Diante do exposto, é possível considerar que o Equus caballus foi selecionado pelas pressões ambientais para perceber as pistas de seus predadores majoritariamente com a visão, pelo fato de ocuparem planícies e serem dotados de um campo de visão panorâmico. Não podem ser descartadas, porém, as contribuições auditivas e olfativas no reconhecimento das pistas dos predadores dos cavalos. Informações dessa natureza são relevantes subsídios que permitem compreender o comportamento inato de fuga dos equinos. Além disso, representam importante contribuição para o aprimoramento do ambiente e do manejo envolvendo os cavalos, evitando-se, assim, estímulos visuais, auditivos e olfatórios que possam amedrontá-los e, consequentemente, causar o empobrecimento de seu bem-estar. São necessários mais estudos focados nas características e pressões ambientais dos predadores dos cavalos para melhor compreender este complexo mecanismo multissensorial dos equinos.

\section{Referências}

Apfelbach R, Blanchard CD, Blanchard RJ, Hayes RA, McGregor IS. The effects of predator odors in mammalian prey species: a review of field and laboratory studies. Neurosci Biobehav Rev. 2005;29(8):1123-44.

Arnould C, Orgeur P, Sempere A, Signoret JP. Répulsion alimentaire chez trois espèces d'ongulés en situation de pâturage : effet des excréments de chien. Rev Ecol-Terre Vie. 1993;48(2):121-32. 
Arnould C, Signoret JP. Sheep food repellents: Efficacy of various products, habituation, and social facilitation. J Chem Ecol. 1993;19(2):225-36.

Beauchamp G. Sleeping gulls monitor the vigilance behaviour of their neighbours. Biol Lett. 2009;5(1): 9-11.

Beauchamp G. What can vigilance tell us about fear? Anim Sent. 2017;15(1):1-31.

Boyd L, Keiper R. Behavioural ecology of feral horses. In: Mills D, McDonnell S (EE.). Behavioural ecology of feral horses. The domestic horse : the origins, developments, and management of its behaviour. Cambridge: Cambridge University Press; 2005. p. 55-82.

Christensen JW, Keeling LJ, Nielsen BL. Responses of horses to novel visual, olfactory and auditory stimuli. Appl Anim Behav Sci. 2005;93(1-2):53-65.

Christensen JW, Malmkvist J, Nielsen BL, Keeling LJ. Effects of a calm companion on fear reactions in naive test horses. Equine Vet J. 2008a;40(1):46-50.

Christensen JW, Rundgren M. Predator odour per se does not frighten domestic horses. Appl Anim Behav Sci. 2008;112(1-2):136-45.

Christensen JW, Zharkikh T, Ladewig J. Do horses generalise between objects during habituation? Appl Anim Behav Sci. 2008b;114(3-4):509-20.

Deecke VB, Ford JKB, Slater PJB. The vocal behaviour of mammal-eating killer whales: communicating with costly calls. Anim Behav. 2005;69(2):395-405.

Forkman B, Boissy A, Meunier-Salaün MC, Canali E, Jones RB. A critical review of fear tests used on cattle, pigs, sheep, poultry and horses. Physiol Behav. 2007;92(3):340-74.

Fureix C, Pagès M, Bon R, Lassalle JM, Kuntz P, Gonzalez G. A preliminary study of the effects of handling type on horses' emotional reactivity and the human-horse relationship. Behav Processes. 2009;82(2):202-10.

Gardner LP. Response of horses to the same signal in different positions. J Comp Psychol. 1937;23(2):305-32.
Ge C, Beauchamp G, LiZ. Coordination and synchronisation of anti-predation vigilance in two crane species. PLoS One. 2011;6(10):e26447.

Hall C. The impact of visual perception on equine learning. Behav Processes. 2007;76(1):29-33.

Hanggi EB. Interocular transfer of learning in horses (Equus caballus). J Equine Vet Sci. 1999;19(8):518-24.

Hanggi EB, Ingersoll JF. Lateral vision in horses: a behavioral investigation. Behav Processes. 2012;91(1):70-6.

Harman AM, Moore S, Hoskins R, Keller P. Horse vision and an explanation for the visual behaviour originally explained by the 'ramp retina'. Equine Vet J. 1999;31(5):384-90.

Hauser MD. Synopsis of the argument. In: Hauser MD. The evolution of communication. Boston: MIT Press; 1996. p. 1-16.

Heffner HE, Heffner RS. Sound localization in large mammals: Localization of complex sounds by horses. Behav Neurosci. 1984;98(3):541-55.

Hess EH. Hess: The natural history of imprinting. Ann N Y Acad Sci. 1972;193(1):124-36.

Keeling LJ, Blomberg A, Ladewig J. Horse-riding accidents: when the human-animal relationship goes wrong. 33rd International Congress of the International Society for Applied Ethology; 17-21 ago 1999; Lillehammer, Noruega. Ås, Noruega: Agricultural University of Norway; 1999.

Leiner L, Fendt M. Behavioural fear and heart rate responses of horses after exposure to novel objects: Effects of habituation. Appl Anim Behav Sci. 2011;131(3-4):104-9.

Lima RAS, Cintra AG. Revisão do Estudo do Complexo do Agronegócio do Cavalo. Brasília: Ministério da Agricultura, Pecuária e Abastecimento; 2016. 56 p. [acesso 9 abr 2017]. Disponível em: https://tinyurl.com/ y9xvgpvr. 
Lorenz K. Evolution and modification of behavior. Chicago: University of Chicago Press; 1965. 121 p.

McGreevy P. Equine Behavior: A Guide for Veterinarians and Equine Scientists Philadelphia: Saunders; 2004. p. 158-60.

Michelena P, Pillot MH, Henrion C, Toulet S, Boissy A, Bon R. Group size elicits specific physiological response in herbivores. Biol Lett. 2012;8(4):537-9.

Miller PE, Murphy CJ. Equine vision. In: Gilger BC (E.). Equine Ophthalmology. St. Louis: Elsevier Saunders; 2010. p. 396-434.

Miller RM. Imprint training of the newborn foal. Colorado: Western Horseman Books; 1991. 144 p.

Mills D, Nankervis K. Comportamento eqüino. São Paulo: Roca; 2005. 213 p.

Paranhos da Costa MJR. Comportamento e Bem-Estar. In: Macari M, Furlan RL, Gonzáles E (EE.). Fisiologia Aviária Aplicada a Frangos de Corte. Jaboticabal: FUNEP/UNESP; 2002. p. 327-45.

Pays 0, Renaud PC, Loisel P, Petit M, Gerard JF, Jarman PJ. Prey synchronize their vigilant behaviour with other group members. Proc Biol Sci. 2007;274(1615): 1287-91.

Pfister JA, Müller-Schwarze D, Balph DF. Effects of predator fecal odors on feed selection by sheep and cattle. J Chem Ecol. 1990;16(2):573-83.

Podgórski T, Jong S, Bubnicki JW, Kuijper DPJ, Churski M, Jędrzejewska B. Drivers of synchronized vigilance in wild boar groups. Behav Ecol. 2016;27(4):1097-103.

Prestwich KN, Brugger KE, Topping M. Energy and communication in three species of hylid frogs: power input, power output and efficiency. J Exp Biol. 1989;144:53-80.

Sato T. Habituação e sensibilização comportamental. Psicol USP. 1995;6(1):231-76.
Schmidek A, Oliveira BN, Trindade P, Paranhos da Costa MJR. Gentle handled foals generalise responses to humans. J Anim Behav Biometeorol. 2018;6(1):1-5.

Spier SJ, Pusterla JB, Villarroel A, Pusterla N. Outcome of tactile conditioning of neonates, or "imprint training" on selected handling measures in foals. Vet J. 2004;168(3):252-8.

Terlouw EMC, Boissy A, Blinet P. Behavioural responses of cattle to the odours of blood and urine from conspecifics and to the odour of faeces from carnivores. Appl Anim Behav Sci. 1998;57(1-2):9-21.

Trindade PHE, Carvalhal MVL, Ceballos MC, Costa FO, Góis KCR, Freitas NR, et al. A preliminary study of novel object test pre-and post-horse cavalcade. 34을 Encontro Anual de Etologia; 12-15 nov 2016; Jaboticabal, SP. Jaboticabal: FUNEP; 2016a [acesso 15 abr 2017]. Disponível em: https://tinyurl.com/y6us9uzz.

Trindade PHE, Góis KCR, Sgarbiero T, Lima MLP, Paranhos da Costa MJR. Conditioning with positive reinforcement for blood sampling in ranch horses. $34^{\circ}$ Encontro Anual de Etologia; 12-15 nov 2016; Jaboticabal, SP. Jaboticabal: FUNEP; 2016b [acesso 15 abr 2017]. Disponível em: https://tinyurl.com/y6us9uzz.

von Borstel UUK, Duncan IJH, Lundin MC, Keeling LJ. Fear reactions in trained and untrained horses from dressage and show-jumping breeding lines. Appl Anim Behav Sci. 2010;125(3-4):124-31.

Waring GH. Horse Behavior. Norwich, NY: William Andrew Publishing; 2003. p. 283-300.

Waring GH. Sounds of the horse (Equus caballus). Bull Ecol Soc Amer. 1971;52(45):330-69.

Warren-Smith AK, McGreevy PD. The use of blended positive and negative reinforcement in shaping the halt response of horses (Equus caballus). Anim Welf. 2007;16(4):481-8.

Yeon SC. Acoustic communication in the domestic horse (Equus caballus). J Vet Behav. 2012;7(3):179-85. 